\title{
TEMPERATURE AND ELECTRIC FIELD DEPENDENCE OF THE VISCOS- ITY OF ELECTRORHEOLOGICAL (ER) FLUIDS: WARMING UP OF AN ELECTRORHEOLOGICAL CLUTCH
}

\author{
SÁNDOR MESTER ${ }^{1}$ AND ISTVÁN SZALAI *1 \\ ${ }^{1}$ Institute of Mechatronics Engineering and Research, University of Pannonia, Gasparich Márk u. 18/A, \\ Zalaegerszeg, 8900, HUNGARY
}

\begin{abstract}
Cognition of the temperature-dependence of intelligent fluids, e.g. electrorheological (ER) and magnetorheological (MR) fluids, is critical for their application. In this paper, the dependence of the viscosity of ER fluids on temperature and electric field strength is examined. A new correlation equation is presented to describe the dependence of the viscosity on temperature by extending the Andrade equation. Considering the dependence of viscosity on the electric field strength and temperature, that equation is used to model the warming up of an ER clutch.
\end{abstract}

Keywords: electrorheological fluid, viscosity, heat effect in ER fluids, ER clutch

\section{Introduction}

Electrorheological (ER) fluids are suspensions made by dispersing micron-sized solid particles with a relative permittivity of $\varepsilon_{\mathrm{p}}$ into a carrier fluid with a smaller relative permittivity of $\varepsilon_{\mathrm{f}}$ [1-3]. Normally, small concentrations of stabilizers are also used to avoid sedimentation. The structure and, therefore, the rheological properties are altered by applying an external electric field. The dispersed particles, guided by the electric field, form chain-like structures [4]. These structures impair the motion of the suspended particles resulting in an increase in the apparent viscosity. Using silicone oil as the carrier fluid is common, although other oils such as transformer oil have also been examined. The dispersed phase can consist of oxides, carbides, etc. ER and magnetorheological (MR) fluids are used in various applications, e.g. couplings, shock dampers, $[5,6]$ ultra-smooth polishing materials, etc. [7].

Most of the applications require the viscosity to be precisely adjusted, however, the viscosity alters as the temperature changes. [8,9] This disadvantage greatly limits its industrial use, as a change can impair fine-tuned systems by creating stern operating conditions. Apart from the need to measure the temperature, the dependence of the parameters of the ER fluids on temperature ought to be considered as well. In addition to the ER effect, an ER fluid is also affected by the thermal motion of particles. This motion works against the ER effect, as it disrupts the chain-like structure [10,11].

*Correspondence: szalai@almos.uni-pannon.hu

\section{Experimental}

The dependence of the viscosity of fluids on the temperature is characterized by a law proposed by Andrade:

$$
\eta=A e^{\frac{B}{T}}
$$

where $\eta$ denotes the dynamic viscosity of the fluid, $T$ stands for the temperature, while $A$ and $B$ represent characteristic constants of the fluid. The constants are experimentally defined for each fluid. For ER fluids, this equation is inadequate because their dependency on the electric field strength is not addressed. In the following chapters, the dependence on the electric field in Eq. 1 is introduced.

\subsection{Samples and viscosity measurements}

To study the thermal and field effects, an Anton Paar Physica MCR 301 rotational rheometer was used to measure the viscosity. Different measuring equipment can be used for MR and ER fluids, moreover, the samples can also be thermostated. The usage of a cylindrical probe is shown schematically in Fig. 1. The length of the probe was $L=40.046 \mathrm{~mm}$, the radius of the probe was $r_{\mathrm{i}}=13.33 \mathrm{~mm}$ and the inner radius of the chamber was $r_{\mathrm{e}}=14.46 \mathrm{~mm}$.

For the measurements, a self-prepared ER fluid was used. The carrier fluid was silicone oil with a viscosity of $1000 \mathrm{mPas}$ at $298 \mathrm{~K}$. The dispersed phase was silica powder of $0.5-10 \mu \mathrm{m}$ in diameter (as the manufacturer 


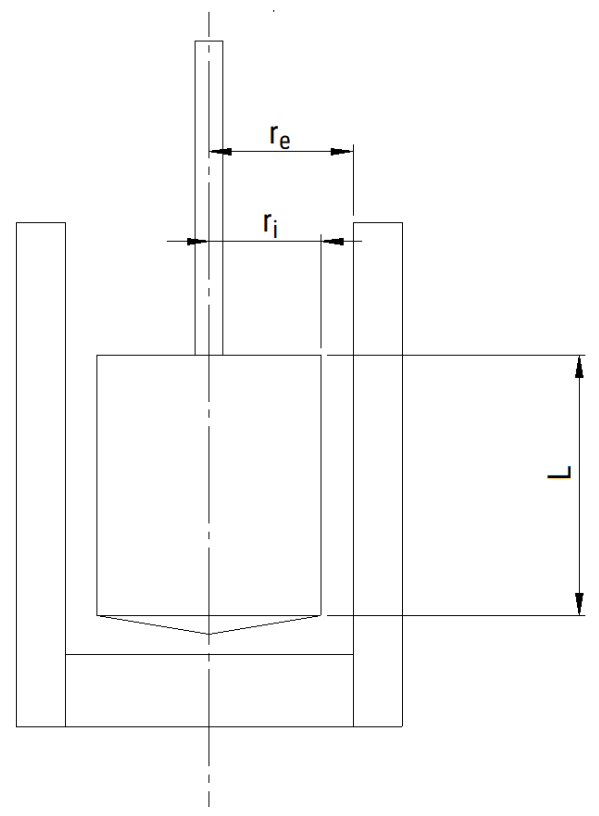

Figure 1: Schematic representation of the probe

claims that $80 \%$ of the particles have diameters of between 1 and $5 \mu \mathrm{m})$. As a result, three different concentrations of particles were investigated, namely 10, 20 and 30 $\mathrm{wt} \%$.

The samples were prepared through a multi-step procedure: after stirring by hand, the fluid was placed in an ultrasonic bath for 15 minutes to mix further. To ensure an air bubble-free ER fluid, the sample was exposed to a vacuum for 10 minutes before being placed in the rheometer.

The samples were measured at six different temperatures at increments of $10 \mathrm{~K}$ ranging from $293 \mathrm{~K}$ to 343 $\mathrm{K}$. Considering the $1.13 \mathrm{~mm}$ gap at the measuring probe, the used voltages resulted in the following electrical field strengths: $0,0.442,0.885,1.327$ and $1.769 \mathrm{MV} / \mathrm{m}$.

The samples were constantly stirred in the rheometer. A two-minute-long stirring cycle in the absence of an electric field came after setting the temperature. A tenminute-long measuring cycle with an electric field was applied and another one-minute-long stirring cycle followed in the absence of an external electric field. The two mixing cycles at the beginning and end ensured that no residual particle arrangements from the previous measurements were present. The viscosity was measured per second.

\section{Measurement Results and Analysis}

\subsection{Temperature dependence of the viscosity}

As an example, the measurement results of an $30 \mathrm{wt} \%$ ER fluid at a temperature of $293 \mathrm{~K}$ are shown in Fig. 2: After an initial rise (while the chain-like structure was forming), the viscosity became roughly constant. The anomalies in the figure were caused by external interferences.

For the analysis, the results of a specific mixing ratio, electric field strength and temperature were averaged into

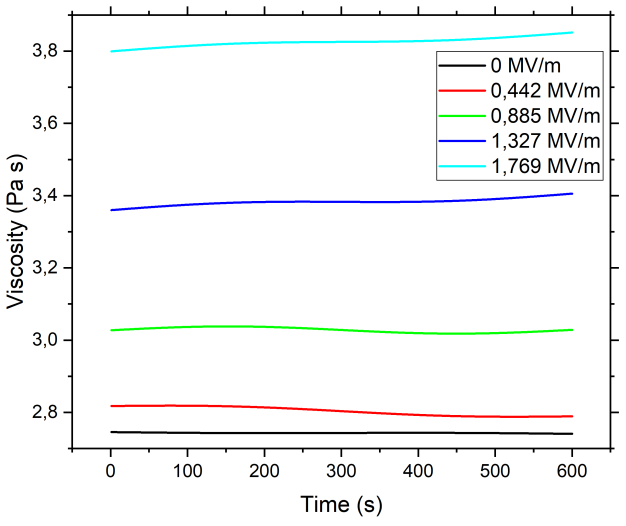

Figure 2: Viscosity measurements under various electric field strengths, $c=30 \%, T=295 \mathrm{~K}$

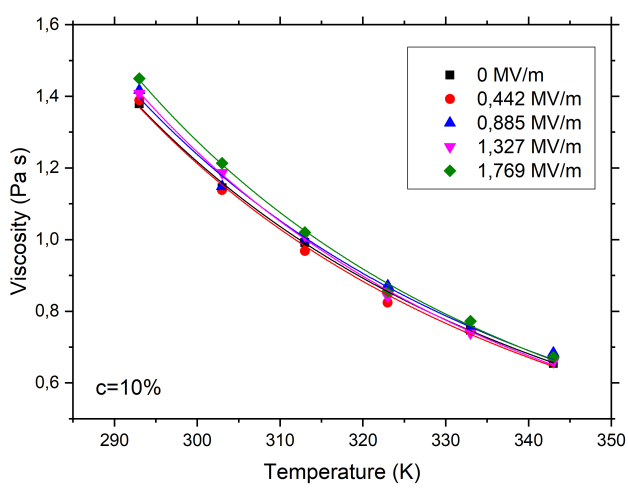

Figure 3: Experimental results and fitted curves (Eq. 2) of viscosity at $c=10 \%$ under various electric field strengths

a single value. The expected tendencies are as follows: as the electric field strength increases, so does the viscosity, while a rise in temperature is inversely proportional to the viscosity.

It was found that Eq. 1 is inappropriate for the exact representation of the dependence of ER viscosity data on temperature, therefore, in terms of the pre-exponential factor, a further temperature dependence was proposed:

$$
\eta=\left(A_{0}+A_{1} T\right) e^{\frac{B}{T}}
$$

where $A_{0}, A_{1}$ and $B$ denote constants derived by fitting Eq. 2 to measurement data.

Figs. 3-5 demonstrate the fitted curves of the different concentrations of ER fluid. The extended formula (Eq. 2) correlates well with the measurements: the worst coefficient of determination for the fittings is $R^{2}=97.3 \%$. Eq. 2 can be used to describe the viscosity of electrorheological fluids as a function of the temperature. 


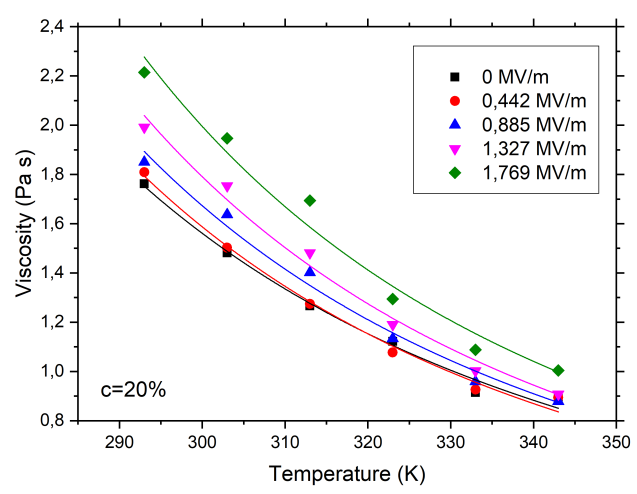

Figure 4: Experimental results and fitted curves (Eq. 2) of viscosity at $c=20 \%$ under various electric field strengths

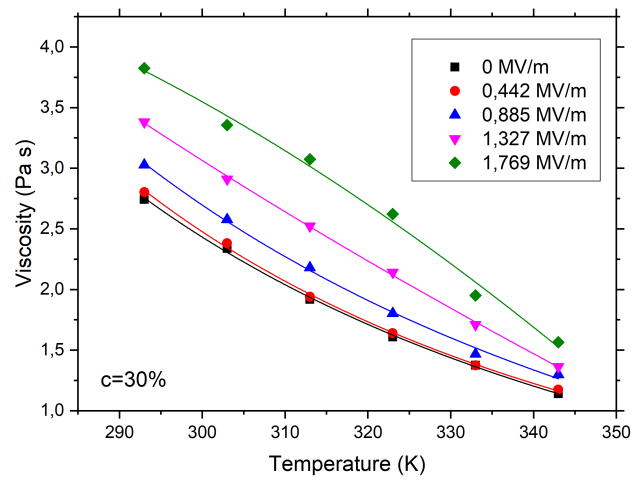

Figure 5: Experimental results and fitted curves (Eq. 2) of viscosity at $c=30 \%$ under various electric field strengths

\subsection{Electric field strength dependence of vis- cosity}

The introduced formula does not concern how the viscosity depends on the electric field strength. In the case of many practical applications, the electric field changes, therefore, the temperature-dependent description of viscosity alone is insufficient.

Eq. 2 can be extended by making the pre-exponential factor dependent on the electric field strength as well. This expansion is carried out via the square of the electric field, indicating that the direction of the field is reversible:

$$
\eta=\left[A_{0}+A_{1} E^{2}+\left(A_{2}+A_{3} E^{2}\right) T\right] e^{\frac{B}{T}}
$$

where $A_{0}, A_{1}, A_{2}, A_{3}$ and $B$ denote constants, while $E$ stands for the electric field strength.

Eq. 3 fitted to the measurement data can be seen in Figs. 6-8. Here the viscosity is represented as a function of the electric field strength and temperature. The coefficients of determination for the fittings are $R_{10 \%}^{2}=$ $99.62 \%, R_{20 \%}^{2}=98.62 \%$ and $R_{30 \%}^{2}=99.06 \%$. Fitting parameters are summarized in Table 1.

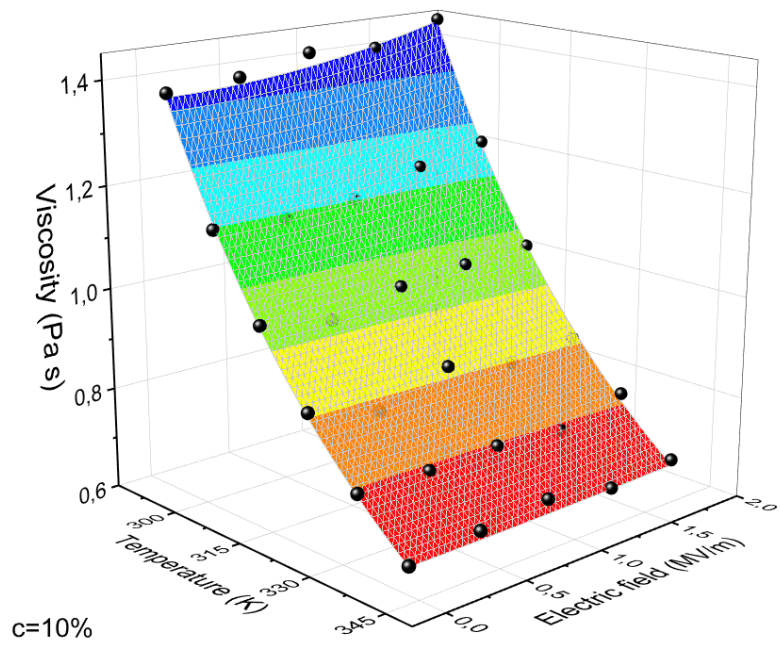

Figure 6: Viscosity measurement data $(\bullet)$ and the fitted surface (Eq. 3) at $c=10 \%$

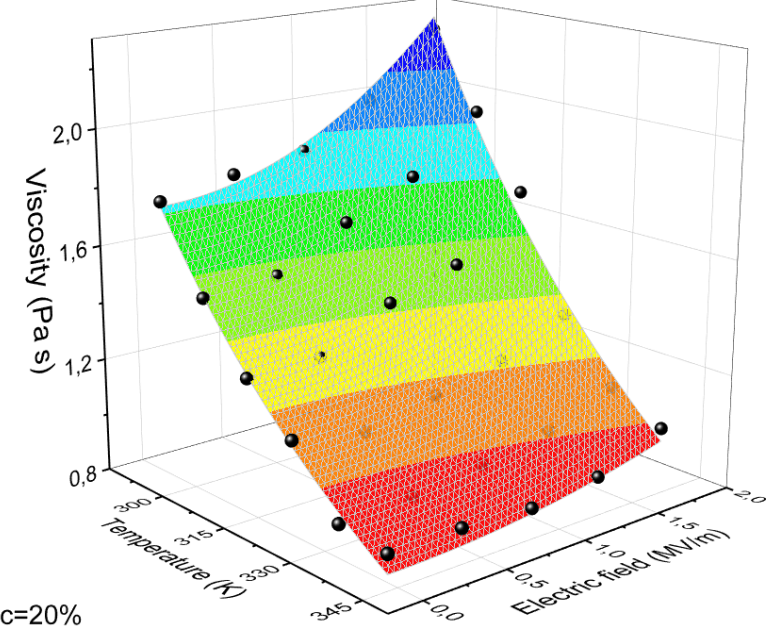

Figure 7: Viscosity measurement data $(\bullet)$ and the fitted surface (Eq. 3) at $c=20 \%$

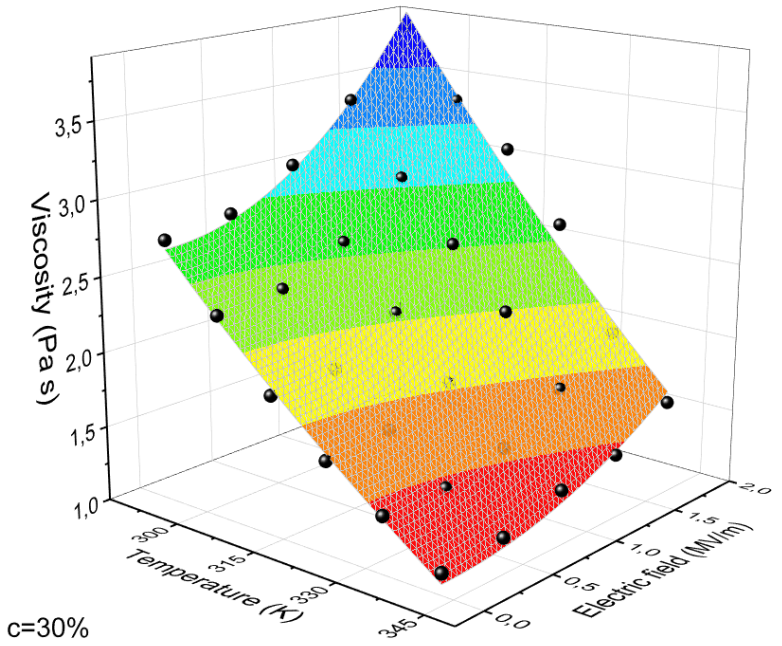

Figure 8: Viscosity measurement data $(\bullet)$ and the fitted surface (Eq. 3) at $c=30 \%$ 
Table 1: Fitted coefficients of Eq. 3 at different ER fluid concentrations

\begin{tabular}{cccc}
\hline \multicolumn{4}{c}{ Concentration $[\mathrm{m} / \mathrm{m}]$} \\
\hline$A_{0}$ & $-2.17623 \mathrm{E}-4$ & 0.12252 & 3.82564 \\
$A_{1}$ & $2.47298 \mathrm{E}-16$ & $2.2336 \mathrm{E}-14$ & $5.10306 \mathrm{E}-13$ \\
$A_{2}$ & $9.7734 \mathrm{E}-6$ & $-2.32975 \mathrm{E}-4$ & $-9.89 \mathrm{E}-3$ \\
$A_{3}$ & $-6.84985 \mathrm{E}-19$ & $-5.8753 \mathrm{E}-17$ & $-1.2747 \mathrm{E}-15$ \\
$B$ & 1831.25188 & 1017.00608 & 3.82564 \\
\hline
\end{tabular}

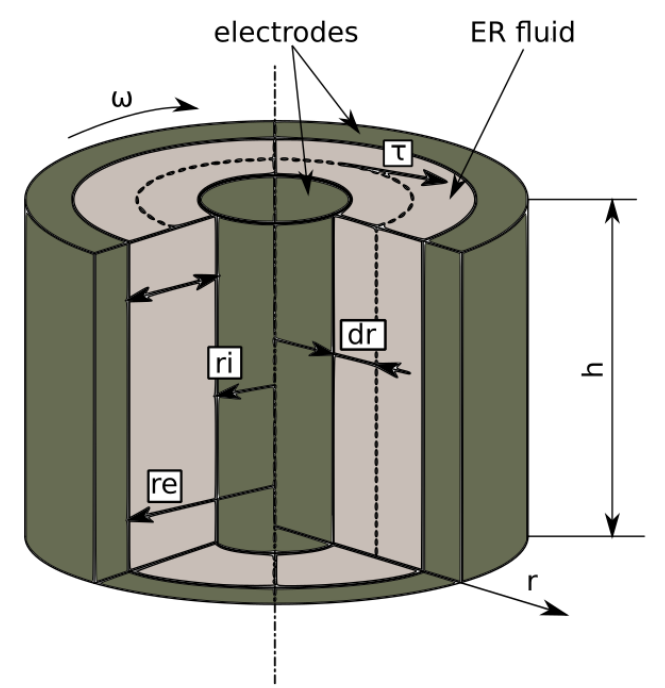

Figure 9: Cylindrical ER clutch model for the dissipation of viscous energy

\section{Temperature rise in an ER clutch}

\subsection{ER clutch model}

A schematic diagram of a simple cylindrical electrorheological clutch is shown in Fig. 9. Nakamura et al. [12] described the temperature rise of similar clutches: their model consisted of several cylinders with gaps between them with radii from $r(i)$ to $r(i+1)$, where $i=1,2 \ldots n$ denotes the number of gaps which are filled with ER fluid with a viscosity of $\eta$. According to the model, assuming the clutch is insulated, the dissipation of the viscous energy per second in the gap $i$ is

$$
d E_{i}=\frac{\partial(\tau A r \omega)}{\partial r} d r
$$

where $A$ denotes the surface of the cylinder, $\tau$ stands for the shear stress and $\omega$ represents the rotational speed. By utilizing the attributes of the cylinders and integrating the formula, the following equation can be derived:

$$
T(t+\Delta t)=\frac{1}{C}\left\{\sum_{i=1}^{n} \frac{2 \pi h \eta(T) \omega^{2} r(i)^{3}}{s} \Delta t\right\}+T(t),
$$

where $T$ denotes the temperature, $s$ stands for the gap, $C$ represents the heat capacity of the fluid, $\omega$ refers to the relative rotational speed and $h$ is the height of the cylinder.

In our system, only one gap is examined so Eq. 5 can be simplified to a differential equation:

$$
\frac{d T}{d t}=\frac{2 \pi h \omega^{2} r^{3}}{s C} \eta(T) .
$$

\subsection{Viscous energy dissipation in the clutch}

Using the model of the ER clutch (Eq. 6), the viscous energy dissipation was calculated by inserting the formula of the viscosity (Eq.3):

$$
\frac{1}{\left[A_{0}+A_{1} E^{2}+\left(A_{2}+A_{3} E^{2}\right) T\right] e^{\frac{B}{T}}} d T=\frac{2 \pi h \omega^{2} r^{3}}{s C} d t .
$$

The integration of the left-hand side of the equation can be solved numerically using mathematical software. Analytical integration requires the expansion of the exponential term into a Taylor series:

$$
\begin{gathered}
\int \frac{e^{-\frac{B}{T}}}{\left[A_{0}+A_{1} E^{2}+\left(A_{2}+A_{3} E^{2}\right) T\right]} d T= \\
=\int \frac{1-\frac{B}{T}+\frac{1}{2 !} \frac{B^{2}}{T}-\frac{1}{3 !} \frac{B^{3}}{T^{3}}+}{\left[A_{0}+A_{1} \cdot E^{2}+\left(A_{2}+A_{3} E^{2}\right) T\right]} d T
\end{gathered}
$$

Eq. 7 can be integrated following the expansion into a Taylor series resulting in the following expression:

$$
\begin{array}{r}
\int_{T_{1}}^{T 2} \frac{1}{\left[A_{0}+A_{1} E^{2}+\left(A_{2}+A_{3} E^{2}\right) T\right] e^{\frac{B}{T}}} d T=\frac{(-1)^{0} B^{0}}{0 !} \frac{1}{a_{0}}\left[\frac{1}{c} \ln (1+c T)\right]_{T_{1}}^{T_{2}}+ \\
+\frac{(-1)^{1} B^{1}}{1 !} \frac{1}{a_{0}}\left[c^{1-1}(-1)^{1} \ln \frac{1+c T}{T}\right]_{T_{1}}^{T_{2}}++\sum_{i=2}^{\infty} \frac{(-1)^{i} B^{i}}{i !} \frac{1}{a_{0}}\left[c^{i-1}(-1)^{i} \ln \frac{1+c T}{T}+\sum_{j=2}^{i} \frac{(-1)^{i-j+1} c^{i-j}}{(j-1) T^{j-1}}\right]_{T_{1}}^{T_{2}}
\end{array}
$$




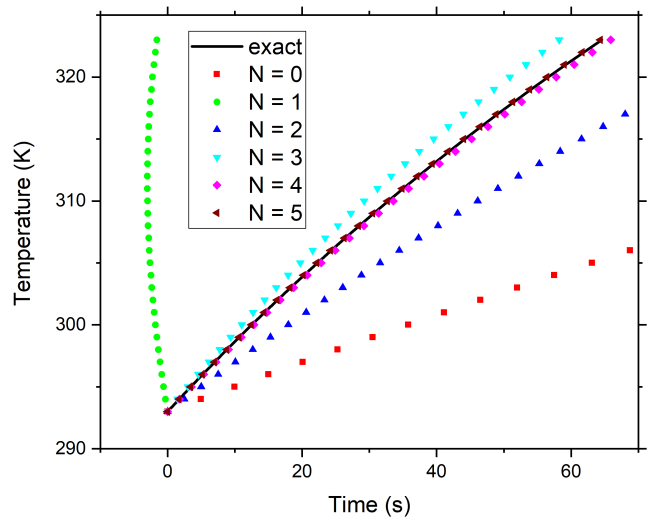

Figure 10: Heating of an ER clutch model as a function of the number of addends, $c=30 \%, E=1.327 \mathrm{MV} / \mathrm{m}$ and $T=295 \mathrm{~K}$

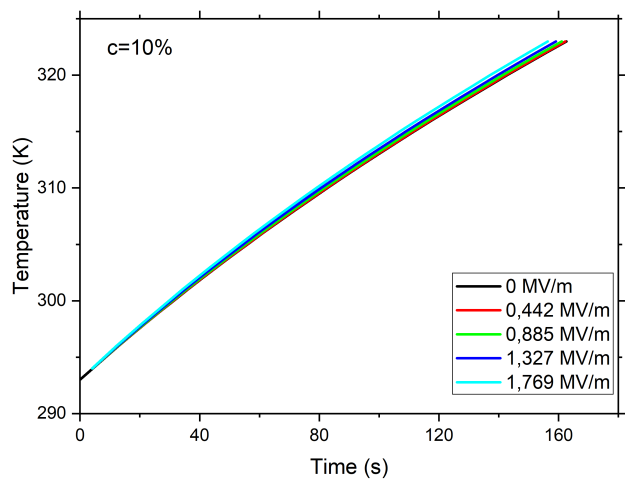

Figure 11: Heating of an ER clutch model over time, $c=$ $10 \%$

where $a_{0}=A_{0}+A_{1} E^{2}$ and $c=\frac{A_{2}+A_{3} E^{2}}{a_{0}}$.

The expression in Eq. 9 can be used to calculate the time needed for the clutch to be heated to a given temperature. The closed formula, a double infinite sum, yields varying results depending on how many terms are used. In the present calculation, the following parameters are used: $h=0.04 \mathrm{~m}, r=0.0133 \mathrm{~m}, s=0.0113 \mathrm{~m}$, $\omega=4.057 \mathrm{rad} / \mathrm{s}$ and $C=0.05 \mathrm{~J} / \mathrm{K}$.

The temperature as a function of the number of addends $N$ is shown in Fig. 10. After the $5^{\text {th }}$ addend, the numerical and analytical solutions are almost identical. To calculate the amount of heating, the physical properties of the Anton Paar probe and the calculated parameters $\left(A_{0}, A_{1}\right.$, etc. $)$ of the examined ER fluids were used.

Figs. 11-13 show the heating results using three different concentrations of ER fluids. As can be observed, as the electric field strength and concentration increase, the temperature also rises faster due to the internal friction.

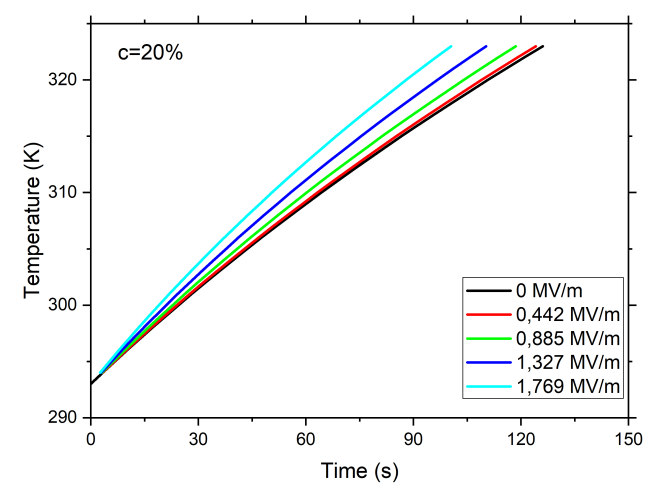

Figure 12: Heating of an ER clutch model over time, $c=$ $20 \%$

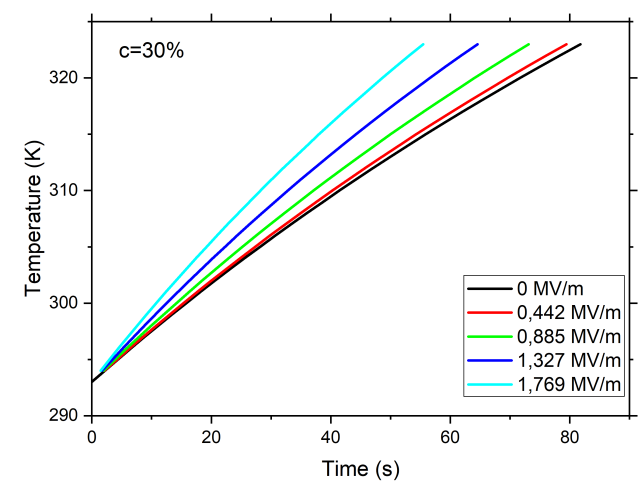

Figure 13: Heating of an ER clutch model over time, $c=$ $30 \%$

\section{Conclusion}

In our paper, a new correlation equation was proposed to describe the dependence of the viscosity of ER fluids on temperature and electric field strength. The proposed equation describes the measurement results with a correspondingly small deviation. The temperature rise of the model system examined by using the aforementioned equations can also form the basis for the description of real systems. The applied model can be further refined, e.g. by taking into account the dependence of heat capacities on temperature.

\section{Acknowledgement}

This research was supported by the European Union and co-financed by the European Social Fund under project EFOP-3.6.2-16-2017-00002.

\section{REFERENCES}

[1] Shin, K.; Kim, D.; Cho, J.-C.; Lim, H. S.; Kim, J. W.; Suh, K. D.: Monodisperse conducting colloidal dipoles with symmetric dimer structure for enhancing electrorheology properties, J. Coll. Interf. Sci., 2012, 374(1), 18-24 DOI: 10.1016/j.jcis.2012.01.055 
[2] Wu, J.; Xu, G.; Cheng, Y.; Liu, F.; Guo, J.; Cui, P.: The influence of high dielectric constant core on the activity of core-shell structure electrorheological fluid, J. Coll. Interf. Sci., 2012, 378(1), 36-43 DOI: 10.1016/j.jcis.2012.04.044

[3] Rankin, P. J.; Ginder, J. M.; Klingenberg, D. J.: Electro- and magneto-rheology, Curr. Op. Coll. Interf. Sci., 1998, 3(4), 373-381 DOI: 10.1016/\$13590294(98)80052-6

[4] Sanchis, A.; Sancho, M.; Martínez, G.; Sebastián, J.; Muñoz, S.: Interparticle forces in electrorheological fluids: effects of polydispersity and shape, Colloid Surf. A, 2004, 249(1-3), 119-122 DOI: 10.1016/j.colsurfa.2004.08.061

[5] Olabi, A. G; Grunwald, A.: Design and application of magneto-rheological fluid, Mat. Design, 2007, 28(10), 2658-2664 DOI: 10.1016/j.matdes.2006.10.009

[6] Bucchi, F.; Forte, P.; Frendo, F.; Musolino, A.; Rizzo, R.: A fail-safe magnetorheological clutch excited by permanent magnets for the disengagement of automotive auxiliaries, J. Int. Mat. Sys. Struc., 2014, 25(16), 2102-2114 DOI: 10.1177/1045389X13517313

[7] Peng, W.; Li, S.; Guan, C.; Li, Y.; Hu, X.: Ultraprecision optical surface fabricated by hydrodynamic effect polishing combined with magnetorhe- ological finishing, Optik, 2018, 156, 374-383 DOI: 10.1016/j.ijleo.2017.11.055

[8] Ayani, M.; Hosseini, L.: The Effect of Temperature and Electric Field on the Behavior of Electrorheological Fluids, in 4th Annual (International) Mechanical Engineering Conference (Isfahan University of Technology, Isfahan, Iran)

[9] Mokeev, A. A.; Gubarev, S. A.; Korobko, E. V.; Bedik, N. A.: Microconvection heat transfer in electrorheological fluids in rotating electric field, J. Phys.: Conf. Ser., 2013, 412, 012008 DOI: 10.1088/1742-6596/412/1/012008

[10] Spaggiari, A.: Properties and applications of Magnetorheological fluids, Frattura ed Integrità Strutturale, 2012, 7(23), 48-61 DOI: 10.3221/IGF-ESIS.23.06

[11] Wang, R.; Wang, Y.C.; Feng, C.Q.; Zhou, F.: Experimental Research on the Heat Transfer Characteristics of Electrorheological Fluid Shock Absorber, in Advances in Industrial and Civil Engineering, Advanced Materials Research, 2012, 594597 (Trans Tech Publications Ltd), 2836-2839 DOI: 10.4028/www.scientific.net/AMR.594-597.2836

[12] Nakamura, T.; Saga, N.; Nakazawa, M.: Thermal Effects of a Homogeneous ER Fluid Device, J. Int. Mat. Sys. Struct., 2003, 14(2), 87-91 DOI: 10.1177/1045389X03014002003 